\title{
Estudo anatômico do xilema secundário da raiz e do caule de Maytenus guyanensis Klotzsch ex Reissek (Celastraceae) ${ }^{1}$
}

\author{
Ressiliane Ribeiro PRATA², Maria Silvia de MENDONÇA ${ }^{3}$
}

\begin{abstract}
RESUMO
Maytenus guyanensis é uma planta medicinal, conhecida popularmente por chichuá, possuindo ação analgésica, antiinflamatória, afrodisíaca e antireumática. O objetivo do presente trabalho foi analisar as características estruturais, da raiz e caule desta espécie como contribuição aos trabalhos anatômicos já realizados para o gênero. O material botânico foi coletado na Reserva Florestal Adolpho Ducke, Manaus/AM onde foram selecionados três indivíduos e de cada um deles retirados fragmentos de $1 \mathrm{~cm}^{3}$ do caule e raiz. Amostras foram seccionadas em micrótomo de deslize e coradas com safranina e azul de astra. A análise estrutural revelou-se de acordo com o registrado pela literatura para o gênero. O xilema secundário da raiz e do caule apresentam parênquima axial apotraqueal, raios multisseriados, heterogêneos, vasos solitários, de distribuição difusa, uniforme, seção circular, com parede delgada, pontoaçōes intervasculares alternas e areoladas.
\end{abstract}

PALAVRas Chave: Chichuá, Plantas medicinais, Lenho, Amazônia.

\section{Anatomic study of secundary xylem of root and stem of Maytenus guyanensis Klotzsch ex Reissek (Celastraceae)}

\begin{abstract}
Maytenus guyanensis, known popularly as chichuá, possess analgesic, anti-inflammatory, aphrodisiac and anti-rheumatic agents. The object of this present wor was the anatomical analysis of material collected of this species at the Adolpho Ducke Forest Reserve. Stem and root fragments of $1 \mathrm{~cm}$ were removed from 3 selected individuals. Sections from the stem and root were cut with slide microtome, and stained with astra blue and safranin. The structural analysis of stems and roots was in accordance with the literature available for the Maytenus genus. The secondary xylem of the root and stem presented parenchyma axial apotracheal, multiseriates, heterogeneous rays, solitary vessels, of diffuse distribution, uniform, circular section, with thin wall, bordered and alternate intervascular pits.
\end{abstract}

KEYWORDS: Chichuá, Medicinal plants, Woody, Amazon. 


\section{INTRODUÇÃO}

Celastrales é constituída por 10 famílias, com espécies predominantemente lenhosas de regióes tropicais e subtropicais (Barroso, 1991). Dentre elas Celastraceae compreende 55 gêneros e aproximadamente 855 espécies, distribuídas nas regiōes tropicais e subtropicais dos dois hemisférios e pouco representadas nas zonas temperadas (Ribeiro et al., 1999; Judd et al., 2002). Maytenus apresenta maior representatividade de espécies na América do Sul em relação ao Continente americano (Gentry, 1993). Atualmente são descritas cerca de 80 espécies para o território brasileiro (Carvalho-Okano \& Leitão Filho, 2005).

As informações estruturais sobre espécies de Maytenus são baseadas nas descriçōes anatômicas elaboradas por Solereder (1908) e Metcalfe e Chalk (1957), nas interpretaçôes ecológicas da anatomia da folha de 11 espécies da África do Sul, dentre elas $M$. oleoides (Hlwatika \& Bhat, 2002), na anatomia da folha de $M$. ardisiaefolia Reiss., $M$. brasiliensis Mart., M. cestrifolia Reiss., M. communis Reiss. e M. obtusifolia Mart. (Joffily \& Vieira, 2005). Além disso, caule e folha das espécies desse gênero são utilizados na medicina tradicional e estruturalmente investigadas com propósitos fitoquímicos e farmacológicos (Duarte \& Debur, 2005). Muitos são os trabalhos que demonstram a atividade antimicrobiana, citotóxica, fitohormonal, antitumoral e antioxidante dos órgãos vegetativos de algumas espécies de Maytenus (Chávez et al., 1997; Gonzalez et al., 2001; González et al., 2001; Pullen et al., 2003; Nuñez et al., 2005).

Maytenus guyanensis é uma árvore de pequeno porte endêmica de terra firme na Amazônia, e conhecida como chichuá, xixuá, chuchahuasi, chucchu huashu, chuchuasi, chuchasha e tonipulmon (Duke \& Vásquez, 1994; Revilla, 2001; Revilla, 2002a; Revilla, 2002b; Borrás, 2003). Suas raízes e caule são utilizados como analgésico, anti-inflamatório, afrodisíaco, relaxante muscular, antireutmático e antidiarréico. A espécie também é indicada no tratamento de artrite, impotência, resfriado, bronquite, hemorróidas, verminoses, lumbago, úlceras externas e usos ginecológicos (Borrás, 2003). Como cosmético é utilizado nas erupçōes cutâneas e previne o câncer de pele (Revilla, 2002a).

Apesar dos seus inúmeros usos, poucos são os trabalhos anatômicos realizados com a raiz e caule de $M$. guyanensis. Estudos recentes foram realizados com casca do caule dessa espécie (Prata, 2007). Para xilema secundário, estudos foram realizados com o caule de espécies de Celastrales por Patel e Bowles (1978), Fernandéz et al. (1998) e Carlquist (2003). Esses estudos utilizaram a anatomia do xilema secundário para fins taxonômicos, sendo apresentadas poucas considerações ecológicas associadas àquele tecido.

O objetivo desse trabalho foi descrever a anatomia do xilema secundário da raiz e do caule de M. guyanensis visando complementar as informaçôes anatômicas existentes para as demais espécies de Maytenus.

\section{MATERIAL E MÉTODOS}

Amostras foram coletadas aleatoriamente do caule e raiz de três indivíduos de $M$. guyanensis na Reserva Florestal Adolpho Ducke, localizada no km 26 da Estrada ManausItacoatiara (AM-010) coordenadas $02^{\circ} 53^{\prime} \mathrm{S}$ e $59^{\circ} 58^{\prime} \mathrm{W}$. O material testemunha encontra-se depositado no Herbário do Instituto Nacional de Pesquisas da Amazônia (INPA), sob o no. 188491 .

Amostras do caule e raiz foram fixadas em formaldeídoácido acético-álcool etílico $50 \%\left(\mathrm{FAA}_{50}\right)$ por 24 horas e preservadas em etanol $70 \%$. Os corpos de prova do lenho foram retirados com dimensão de $1 \mathrm{~cm}^{3}$, orientados, amolecidos e secionados em micrótomo de deslize. As secçōes foram preparadas de acordo com as técnicas usuais de microscopia de luz, coradas com safranina e azul de astra (somente seções da raiz) e montadas em meio sintético (Johansen, 1940).

Fragmentos do caule foram submetidos ao processo de maceração pelo método de Jeffrey a base de ácido crômico $10 \%$ e ácido nítrico $10 \%$ (1:1), corados com safranina e montados em gelatina glicerinada (Johansen, 1940).

A estrutura anatômica foi analisada com o auxílio de microscópio Olympus CX40, e máquina fotográfica digital Lumix, Panasonic, do Laboratório de Citologia, Departamento de Morfologia da Universidade Federal do Amazonas (UFAM), e também com o auxílio do fotomicroscópio Olympus AX70 do Laboratório de Anatomia Vegetal do Departamento de Biologia Vegetal da Universidade Federal de Viçosa (UFV).

As medidas de espessura de parede, diâmetro e comprimento de elemento de vaso, largura e comprimento de raios, foram alcançadas com o auxílio do Programa SigmaScan Image Analysis, Versão 3.0. A terminologia adotada para a análise anatômica segue aquela estabelecida pelo IAWA Committee (1989).

\section{RESULTADOS}

RAIZ

Camadas de crescimento distintas, delimitadas por faixas de parênquima marginal, distribuindo-se em intervalos regulares. Vasos de distribuição difusa, uniforme, secção circular, com parede delgada (cerca de 2,45 $\mu \mathrm{m}$ de espessura) e poros solitários (diâmetro tangencial de 20-29 $\mu \mathrm{m}$ ) (Figura $1 \mathrm{~A})$.

Elementos de vaso com e sem apêndices, quando presentes se encontram em ambas as extremidades dos elementos 
condutores. As pontoações intervasculares são alternas e areoladas.

Parênquima axial apotraqueal em seção transversal em faixas (Figura 1A). Raios multisseriados, com extremidades unisseriadas (Figura 1B), heterogêneos, compostos por células eretas marginais e centrais procumbentes, com 3 a 5 células de largura, predominando os de 3 células. A altura dos raios varia de 12 a 23 células, predominando os de 22 células com cerca de 259,61 $\mu \mathrm{m}$ de altura (Figura 1C). Fibras libriformes.

\section{CAULE}

Camadas de crescimento distintas, delimitadas por faixas de parênquima marginal, distribuindo-se em intervalos regulares (Figura 2A).

Vasos solitários são predominantes, com arranjo difuso uniforme (Figura 2A), parede delgada (cerca de 2,50 $\mu \mathrm{m}$ de espessura) e diâmetro tangencial de 26-29 $\mu \mathrm{m}$. Elementos de vasos longos (com cerca de 494,5 $\mu \mathrm{m}$ de comprimento). Elementos de vaso com apêndices em ambas as extremidades (Figura 2D). As pontoações intervasculares são alternas e as placas de perfuração são simples.

Parênquima apotraqueal difuso, com parênquima axial em faixas marginais com mais de 4 células de largura (Figura 2A).

Raios predominantemente multisseriados com 2 a 3 células de largura, predominando os de 2 células. Altura dos raios de 15 a 28 células, predominando os de 17 e 25 células (Figura 2B). Raios unisseriados são raros, com 8 a 12 células de altura; os heterogêneos possuem células retas nas margens e centrais procumbentes (Figuras 2C). Cristais prismáticos de oxalato de cálcio no parênquima radial (Figura 2B). Fibras libriformes.

\section{DISCUSSÃO}

Para espécies arbóreas de áreas alagáveis (várzea e igapó) na Amazônia, confirmou-se que a formação de camadas de crescimento do xilema secundário está associada aos pulsos de inundação (Worbes, 1989, 1995). Embora não se possa comprovar tal associação para $M$. guyanensis, pois esta espécie é endêmica de área não alagável da Amazônia (terra firme), ela apresenta camadas de crescimento bem delimitadas, característica comum para as espécies de Celastraceae (Metcalfe \& Chalk, 1957; Duarte \& Debur, 2005). Dessa forma, estudos dendrocronológicos entre espécies da Amazônia de áreas alagáveis ou não alagáveis, são necessários para investigar quais os fatores abióticos que determinam a formação de camadas de crescimento.

$\mathrm{O}$ arranjo difuso uniforme dos vasos entre as angiospermas tropicais é o mais freqüente. Os vasos solitários como observados em $M$. guyanensis são comuns em regiōes de clima homogêneo (Alves \& Angyalossy-Alfonso, 2000). Essa característica dos vasos está relacionada ao xilema secundário de espécies que ocorrem em regiōes onde não há oscilações climáticas, como é o caso da Amazônia, onde as amplitudes térmicas e a umidade relativa do ar não apresentam alteraçóes bruscas ao longo do ano.

Algumas outras características do lenho de diferentes espécies estão relacionadas a fatores ambientais. De acordo com Angyalossy \& Alves (2005) uma destas características refere-se ao diâmetro dos vasos.

Vasos de maior diâmetro são comuns para espécies de ambiente quente e úmido (Angyalossy \& Alves, 2005). Embora o diâmetro dos vasos de $M$. guyanensis contrarie as evidências apresentadas para plantas de ambiente quente e úmido, uma vez que estes apresentam diâmetro de vaso menor em relação ao padrão descrito por Angyalossy e Alves (2005), as placas de perfuração dessa espécie são simples. Essa característica confere uma maior eficiência na condução de água e íons (Esau, 1976). Associando-se as características de diâmetro de vaso e tipos de placas de perfuração, sugerese que elementos de vaso com placas de perfuração simples representam melhor as condições fisiológicas de eficiência na condução de água em ambientes úmidos, não importando dessa maneira o maior ou menor diâmetro dos vasos.

Embora Metcalfe \& Chalk (1957) mencionem que os vasos das raízes de representantes de Celastraceae possuam frequentemente tilos, eles não foram observados nos vasos da raiz de $M$. guyanensis. A presença e a velocidade de formação dos tilos geralmente têm relação com os mecanismos de resistência a patógenos vasculares (Pascholati \& Leite, 1995). Em relação à condução do xilema, o significado dos tilos não é compreendido com clareza. Entretanto, em elementos de vasos cujo diâmetro é inferior a $80 \mu \mathrm{m}$ como em $M$. guyanensis, é reportado na literatura que não ocorre formação de tilos (Costa et al., 2006).

Parênquima axial abundante é uma característica do xilema secundário de plantas de regiōes tropicais, de baixas latitudes como Norte e Nordeste do Brasil (Wheeler \& Baas, 1991; Alves \& Angyalossy-Alfonso, 2002). Em M. guyanensis há abundância de parênquima axial corroborando os resultados desses autores. Além disso, células do parênquima axial e do raio estão associadas ao acúmulo e mobilização de metabólitos, atuando como uma possível adaptação às condiçōes em que as taxas fotossintéticas são altas.

Nas raízes e caule com espessamento secundário, o amido é acumulado geralmente nas células parenquimáticas, explicando assim a razão da raiz possuir uma proporção acentuada de tecido parenquimático em relação ao caule, cuja função metabólica principal é acúmulo de substâncias ergásticas (Fernandéz et al.,1998; Costa et al., 2006). Estas observações podem ser comprovadas em caule e raiz de $M$. guyanensis. Compostos fenólicos, cristais e outras substâncias 
de reserva também são frequentemente encontrados nas células de parênquima do xilema secundário. A formação de cristais de oxalato de cálcio, como observado para $M$. guyanensis, é uma das formas de imobilização de cálcio nos tecidos das plantas, e um meio de evitar acúmulo de oxalato tóxico, atuando no suporte mecânico e proteção contra herbivoria (Franceschi \& Horner Jr., 1980).

Além dessas considerações, as características do xilema secundário existentes para Celastrales reforçam os resultados de filogenética desse grupo de plantas. Patel \& Bowles (1978) relataram para Pennata corymbosa (Icacinaceae) a presença de poros difusos, vasos solitários com placa de perfuração escalariforme, parênquima axial moderadamente abundante
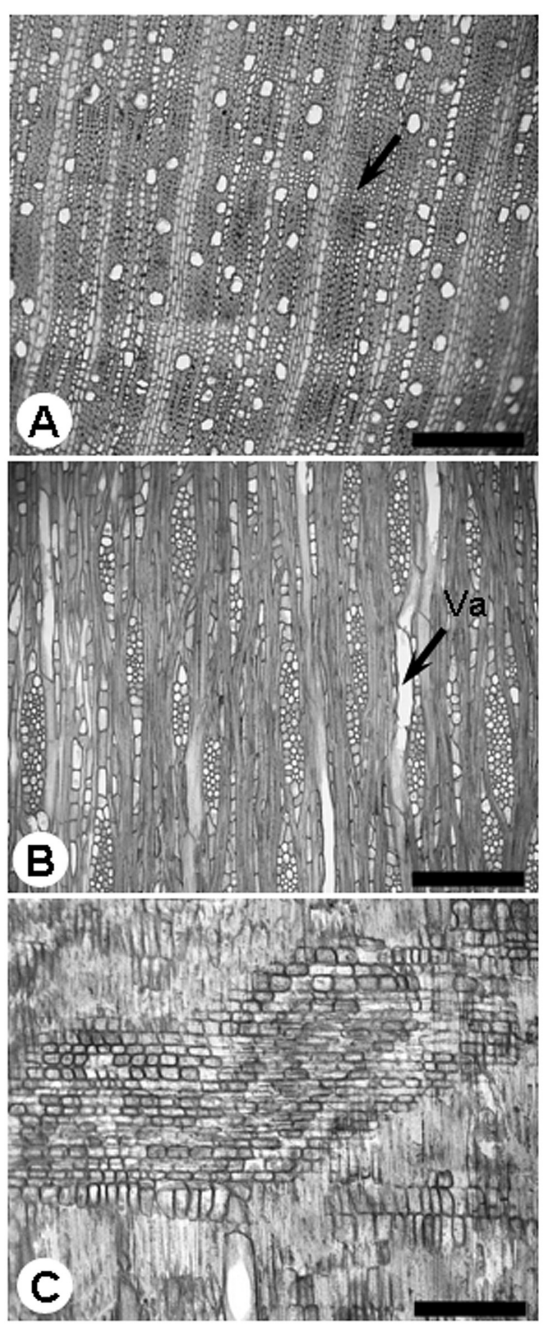

Figura 1 - Seções da raiz em crescimento secundário de Maytenus guyanensis. A. Seção transversal. Camadas de crescimento (seta). Porosidade difusa. Vasos solitários. B. Seção tangencial. Raios heterogêneos multisseriados. C. Seção radial. Raios heterogêneos. Va- vaso. Bar $=50 \mu \mathrm{m}$. a abundante, difuso, difuso em agregados e paratraqueal escasso, e raios heterogêneos. Carlquist (2003) investigou a anatomia da madeira de Aextoxicaceae para a inclusão desta em Berberidopsidales, mencionando para Aextoxicon punctatum presença de traqueídes, maioria de elementos de vaso múltiplos, traqueídes longos e raios heterogêneos. Fernandéz et al. (1998) descreveram xilema secundário do caule e da raiz com porosidade difusa, vasos solitários, parênquima paratraqueal escasso e apotraqueal difuso em agregados e raios multisseriados para Hippocratea excelsa (Hippocrateaceae).

A anatomia do xilema secundário de $M$. guyanensis (Celastraceae) apresentou maior similaridade à de Hippocratea excelsa (Hippocrateaceae). Essa similaridade entre caracteres reafirma o posicionamento taxonômico de Hippocrateaceae nos sistemas de classificação de angiospermas propostos nos últimos anos (Takhtajan, 1997; Judd et al., 2002; APG, 2003). Além disso, os resultados deste trabalho corroboram as descriçōes existentes para xilema secundário de espécies de Celastrales, bem como as considerações ecológicas para espécies distribuídas em ambientes homogêneos de temperatura e umidade.
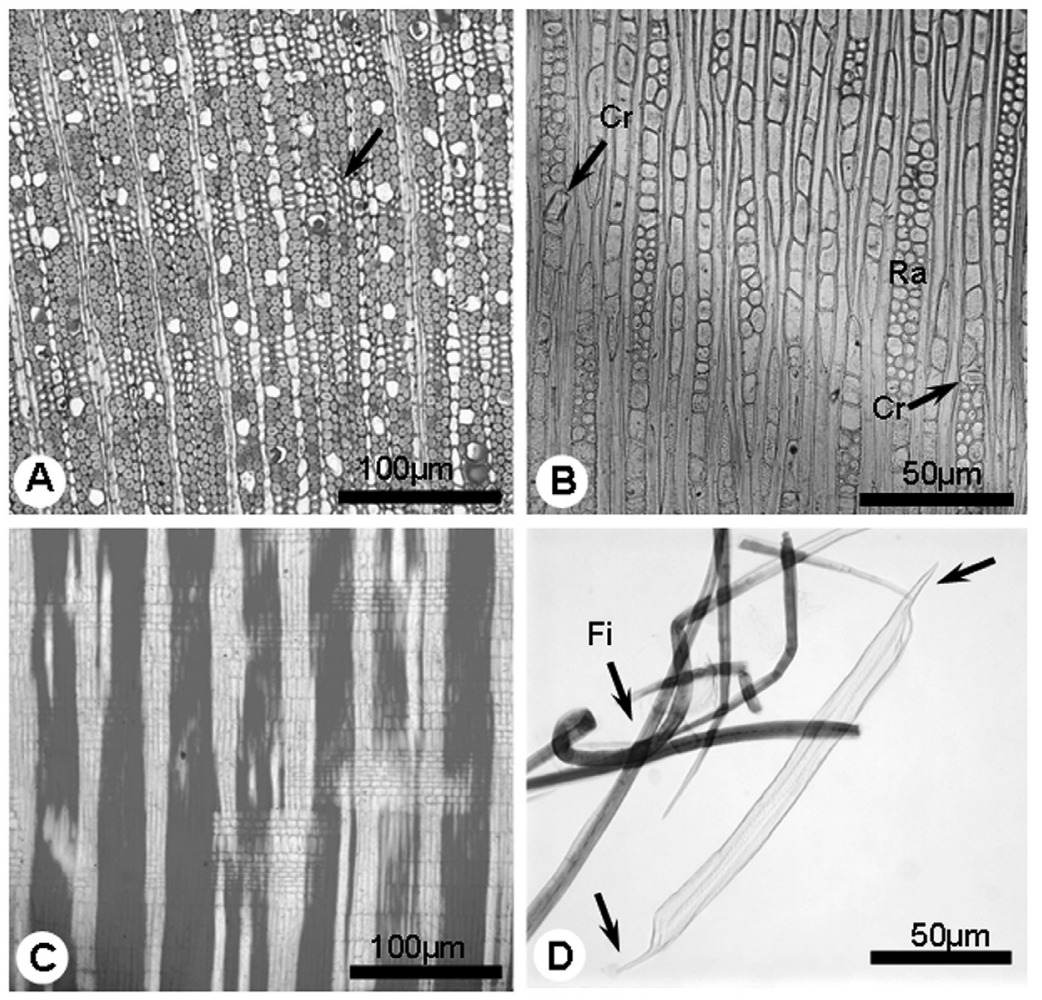

Figura 2 - Estrutura do caule de Maytenus guyanensis. A. Seção transversal. Camadas de crescimento (seta). Porosidade difusa. Parênquima axial difuso. Vasos solitários. B. Seção tangencial. Monocristais prismáticos (Cr). C. Seção radial. Raio multisseriado heterogêneo. D. Fibras (Fi) e elementos de vaso com apêndices e placas de perfuração simples (setas). Bar=A,C $100 \mu \mathrm{m} ; \mathrm{B}, \mathrm{D} 50 \mu \mathrm{m}$. 


\section{AGRADECIMENTOS}

Ao MSc. Tarcísio, MSc. Duncan e ao Dr. Oscar da Universidade Federal do Amazonas. Ao Conselho Nacional de Desenvolvimento Científico e Tecnológico (CNPq), pela bolsa concedida a primeira autora. Ao suporte financeiro concedido pela Fundação de Amparo à Pesquisa do Estado do Amazonas (FAPEAM).

\section{BIBLIOGRAFIA CITADA}

Alves, E.S.; Angyalossy-Alfonso, V. 2000. Ecological trends in the wood anatomy of brazilian species.1. Growth rings and vessels. IAWA Journal, 21(1): 3-30.

Alves, E.S.; Angyalossy-Alfonso, V. 2002. Ecological trends in the wood anatomy of some brazilian species. 2. Axial parenchyma, rays and fibres. IAWA Journal, 23(4): 391-418.

Angyalossy, V; Alves, E. S. 2005. Madeiras utilizadas na fabricação de arcos para instrumentos de corda: aspectos anatômicos. Acta Botanica Brasilica, 19(4): 819-834.

APG (The Angiosperm Phylogeny Group). 2003. An update of the Angiosperm Phylogeny Group classification for the orders and families of flowering plants: APG II. Botanical Journal of the Linnean Society, 141: 399-436.

Barroso, G.M. 1991. Sistemática de angiospermas do Brasil. Vol. 2. Editora UFV, Viçosa. 326pp.

Borrás, M.R.L. 2003. Plantas da Amazônia: medicinais ou mágicasPlantas comercializadas no Mercado Municipal Adolpho Lisboa. Editora Valer/Governo do Estado do Amazonas, Manaus. 322pp.

Carlquist, S. 2003. Wood anatomy of Aextoxicaceae and Berberidopsidaceae is compatible with their inclusion in Berberidopsidales. Systematic Botany, 28(2): 317-325.

Carvalho-Okano, R.M.; Leitão Filho, H.F. 2005. O gênero Maytenus Mol. emend. Mol. (Celastraceae) no Brasil extra-amazônico. In: M.S. Reis; S.R. Silva (Eds). Conservação e uso sustentável de Espinheira Santa, 1: 11-51.

Chávez, H.; Estévez-Braun, A.; Ravelo, A.G.; González, A.G. 1997. First exemples of dammarane triterpenes isolated from Celastraceae. Tetrahedron, 53(18): 6465-6472.

Costa, C.G.; Callado, C.H.; Coradin, V.R.; Carmelo-Guerreiro, S.M. 2006. Xilema. In: Appezzato-da-Glória, B.; CarmelloGuerreiro, S.M. (Eds). Anatomia vegetal. 2a ed. Editora UFV, Viçosa. p. 129-154.

Duarte, M.R.; Debur, M.C. 2005. Stem and leaf morphoanatomy of Maytenus ilicifolia. Fitoterapia, 76: 41-49.

Duke, J.A.; Vasquez, R. 1994. Amazonian ethnobotanical dictionary. CRC Press, Boca Raton, Florida, USA. p. 114.

Esau, K. 1976. Anatomia das plantas com sementes. Edgard Blücher, São Paulo. 293pp.

Franceschi, V.R.; Horner Jr., H.T. 1980. Calcium oxalate crystals in plants. The Botanical Review, 46: 361-427.
Fernandéz, M. G. V.; Morales, J. B.; Ângeles, G. 1998. Estudio anatómico de Hippocratea excelsa HBK. (Hippocrateaceae). Acta Botánica Mexicana, 43: 7-21.

Gentry, A.H. 1993. A field guide to the families and genera of woody plants of Northwest South America (Colombia, Ecuador, Peru) with supplementary notes on herbaceous taxa. Conservation International, Washington. 895pp.

González, A.G.; Kennedy, M.L.; Rodríguez, F.M.; Bazzocchi, I.L.; Jiménez, I.A.; Ravelo, A.G.; Moujir, L. 2001. Absolute configuration of triterpene dimmers from Maytenus species (Celastraceae). Tetrahedron, 57: 1283-1287.

Gonzalez, F.G.; Portela, T.Y.; Stipp, E.J.; Di Stasi, L.C. 2001. Antiulcerogenic and analgesic effects of Maytenus aquifolium, Sorocea bomplandii and Zolernia ilicifolia. Journal of Ethnopharmacology, 77: 41-47.

Hlwatika, C. N. M.; Bhat, R. B. 2002. An ecological interpretation of the difference in leaf anatomy and its plasticity in contrasting tree species in Orange Kloof, Table Mountain, South Africa. Annals of Botany, 89: 109-114.

IAWA Committee. 1989. International association of wood anatomists. List of microscopic features for hardwood identification. IAWA Bulletin, 10: 220-332.

Joffily, A.; Vieira, R. C. 2005. Anatomia foliar de Maytenus Mol. emend Mol. (Celastraceae), ocorrente no Estado do Rio de Janeiro, Brasil. Acta Botanica Brasilica, 19(3): 549-561.

Johansen, D.A. 1940. Plant microtechnique. Mcgraw-Hill Book (Co.), New York. 523pp.

Judd, W.S.; Campbell, C.S.; Kellogg, E.A.; Stevens, P.F.; Donoghue, M.J. 2002. Plant systematics: a phylogenetic approach. 2aed. Sinauer, New York. 576pp.

Metcalfe, C.R.; Chalk, L. 1957. Anatomy of the dicotyledons: leaves, stems and wood in relation taxonomy with notes in economic uses. Vol. 2. Clarendon Press, Oxford. p.387-397.

Nuñez, M.J.; Reyes, C.P.; Jiménez, I.A.; Moujir, L.; Bazzocchi, I. L. 2005. Lupane triterpenoids from Maytenus species. Journal of Natural Products, 68(7): 1018-1021.

Pascholati, S.F.; Leite, B. 2005. Hospedeiro: mecanismo de resistência. In: Bergamin Filho, A.; Kimati, H.; Amorin, L (Eds). Manual de fitopatologia: princípios e conceitos. Vol.1. $3^{\mathrm{a}} \mathrm{ed}$. Ceres, São Paulo. p. 417-453.

Patel, R. N.; Bowles, A. 1978. Wood anatomy of the dicotyledons indigenous to New Zealand 12. Icacinaceae. New Zealand Journal of Botany, 16: 7-12.

Prata, R. R. 2007. Aspectos anatômicos e etnofarmacológicos do caule e raiz de Maytenus guyanensis Klotzsch ex Reissek (Celastraceae). Dissertação de Mestrado, Instituto Nacional de Pesquisas da Amazônia/Universidade Federal do Amazonas, Manaus, Amazonas. 80pp.

Pullen, C.B.; Schmitz, P.; Hoffmann, D.; Meurer, K.; Boettcher, T.; Bamberg, D.V.; Pereira, A.M.; França, S. de C.; Hauser, M.; Geertsema, Wyk, A.V.; Mahmud, T.; Floss, H. G.; Leistner, E. 2003. Occurrence and non-detectability of maytansinoids in individual plants of the genera Maytenus and Putterlickia. Phytochemistry, 62: 377-387. 
Revilla, J. 2001. Plantas da Amazônia - oportunidades econômicas e sustentáveis. SEBRAE-AM/INPA, Manaus. 405pp

Revilla, J. 2002a. Apontamentos para a cosmética amazônica. SEBRAE-AM/INPA, Manaus.532pp.

Revilla, J. 2002b. Plantas úteis da bacia amazônica. SEBRAE-AM/ INPA, Manaus. 445pp.

Ribeiro, J. E. L. S.; Hopkins, M. J. G.; Vicentini, A.; Sothers, C.A.; Costa, M.A.S.; Brito,J. M.; Souza,M.A.D.; Martins,L.H.; Lohmann, L. G.; Assunção, P.A.; Pereira,E. C.; Silva, C. F.; Mesquita, M. R.; Procópio, L. C. 1999. Flora da Reserva DuckeGuia de identificação das plantas vasculares de uma floresta de terra firme na Amazônia Central. INPA-DFID, Manaus. 800 pp.

Solereder, H. 1908. Systematic anatomy of the dicotyledons II. Clarendon Press, Oxford. p. 874-880.
Takhtajan, A. 1997. Diversity and classification of flowering plants. Columbia University Press, New York. 643pp.

Wheeler, E.A.; P. Baas. 1991. A survey of the fossil record for dicotyledonous wood and its significance for evolutionary and ecological wood anatomy. IAWA Bulletin, n.s. 12: 275-332.

Worbes, M. 1989. Growth rings, increment and age of trees in inundation forests, savannas and a mountain forest in the Neotropics. IAWA Bulletin, n.s. 10: 109-122.

Worbes, M. 1995. How to measure growth dynamics in tropical trees - A review. IAWA Journal, 16: 337-351.

Recebido em 11/06/2007

Aceito em 15/01/2009 